\title{
Post-Translational Modification of
} Adiponectin Affects Lipid Accumulation, Proliferation and Migration of Vascular Smooth Muscle Cells

\author{
Xinzhong Chen ${ }^{\mathrm{a}}$ Yanhong Yuan ${ }^{\mathrm{a}}$ Qin Wang ${ }^{\mathrm{a}} \quad$ Fei Xie $^{\mathrm{a}} \quad$ Dongsheng Xia $^{\mathrm{b}}$ \\ Xianguo Wang ${ }^{c}$ Yutao Wei ${ }^{a}$ Ting Xie ${ }^{d}$ \\ aDepartment of Cardiovascular Surgery, Union Hospital, Tongji Medical College, Huazhong University \\ of Science and Technology, Wuhan, bDepartment of Cardiovascular Surgery, Henan Provincial People's \\ Hospital, Zhengzhou, 'Department of Cardiothoracic Surgery of Zhongnan Hospital of Wuhan \\ University, Wuhan, dDepartment of Cardiac Surgery, HaiNan Provincial People's Hospital, Hainan, China
}

\section{Key Words}

Apn • Glycosylation • Atherosclerosis • Human vascular smooth muscle cells

\begin{abstract}
Background/Aims: Adiponectin (Apn) is a multifunctional adipokine that circulates as several oligomeric complexes in the blood stream. Previous reports showed that several conserved lysine residues within the $\mathrm{N}$-terminal collagenous domain of Apn are modified by hydroxylation and glycosylation. Here, we investigated the potential roles of post-translational modifications of Apn on the function of human vascular smooth muscle cells (VSMCs). Methods: Blood samples of 92 coronary artery disease (CAD) patients and 20 healthy volunteers were collected and total and high molecular weight (HMW) Apn concentration and glycosylation were analyzed. Results: The results revealed that total and HMW Apn derived from blood samples of CAD patients with severe stenosis significantly increased, however the glycosylation of HMW Apn significantly decreased. Functional studies of human VSMCs revealed that glycosylated Apn significantly inhibited the oxidized LDL-induced lipid accumulation, proliferation and migration of VSMCs, whereas non-glycosylated Apn had no inhibitory effects. Conclusion: Taken together, these data suggest that glycosylation of Apn is critically involved in regulating function against atherosclerosis by inhibiting lipid accumulation and proliferation and migration of VSMCs.

\section{Introduction}

Adiponectin (Apn), which is secreted by adipose tissue, accounts for about $0.01 \%$ of the total serum protein and is recognized as an important hormone which links with cardiovascular disease (CVD). Apn secreted into the circulation as several oligomeric 
complexes such as low molecular weight (LMW), medium molecular weight (MMW) and high molecular weight (HMW) multimers, is an adipokine with multiple beneficial effects. Extensive post-translational modifications including hydroxylation and glycosylation are required for efficient Apn multimerization, secretion and function [1, 2]. Structural analyses revealed that the basic subunit is made up of a collagenous $\mathrm{N}$-terminal domain, and a C-terminal globular head and these subunits associate to form a trimer (LMW complex), sulfhydryl-dependent hexamers (MMW complex) and high molecular weight multimers (HMW complex), which are stable in vitro and in vivo [3]. The activity of HMW Apn is stronger than those of LMW and MMW forms. A number of studies have investigated the role of posttranslational modifications in the assembly of the HMW isoform. The results showed that the collagenous region of Apn produced in live cells is both hydroxylated and glycosylated with a glucosylgalactosyl moiety on four conserved lysines [4, 5], which is different from the linear Apn produced in E.coli. In addition, mutations of Apn have been reported in diabetic patients in whom the protein show impaired multimerization [6].

Apn is recognized as an important hormone which links with obesity, insulin resistance,cancer and atherosclerosis (AS) [7, 8]. In human cancer, Apn is known to suppress cancer vascularization $[9,10]$. Proliferation and migration of vascular smooth muscle cell (VSMCs) is one of important mechanisms in AS [11]. Apn is known to suppress vascular oxidative stress and has an anti-atherogenic potential [12]. In prospective studies, increased serum HMW Apn has been associated with lower risk of developing cardiovascular diseases [13]. However, the data about the predictive value of Apn in coronary artery disease (CAD) are conflicting $[14,15]$; that is, increase of HMW Apn may be associated with a poor prognosis for advanced disease states such as ischemic heart failure [16]. Therefore, we speculate that the post-translation of Apn may play an important role in its circulating levels and function in CAD.

In order to undersand the potential roles of post-translational modifications of Apn in $\mathrm{CAD}$, the present experiment was designed to analyze the correlation of glycosylation of Apn with the severity of CAD and glycosylated Apn on the lipid accumulation, proliferation and migration of VSMCs. We found, although HMW Apn increased, glycosylation of Apn significantly decreased in the blood sample derived from CAD patients. Functional studies of VSMCs revealed that glycosylated Apn significantly inhibited the oxidized LDLinduced lipid accumulation, proliferation and migration of VSMCs, whereas non-glycosylated Apn had no inhibitory effects. Taken together, these data suggest that glycosylation is critically involved in function of Apn.

\section{Materials and Methods}

\section{Patients}

This study comprised 92 patients with CAD who were consecutively recruited at the Department of Cardiovascular Surgery, Union Hospital, Huazhong University of Science and Technology between January 2015 and January 2016 and 20 healthy volunteers. All patients had anginal symptoms and electrocardiographic changes consistent with ischemia. All angiography results were evaluated by two experienced cardiologists who were blinded of the clinical characteristics of the patients. The severity of the CAD was evaluated using angiographic Gensini scores (GS) [17]. GS is a highly accepted scoring system for evaluating the coronary atherosclerotic burden. In this scoring system, grading is primarily done according to the narrowing of the lumen of the coronary arteries. For $1 \%$ to $25 \%$ lumen stenosis 1 point is given, $26 \%$ to $50 \%$ lumen stenosis 2 points, $51 \%$ to $75 \%$ lumen stenosis 4 points, $76 \%$ to $90 \%$ lumen stenosis 8 points, $91 \%$ to $99 \%$ lumen stenosis 16 points and for complete stenosis 32 points. The obtained scores are then multiplied by coefficients representing the importance of the coronary vessel and the segment in which stenosis is present. For the left main coronary artery a coefficient of 5 is used, for the left anterior descending and proximal part of the circumflex coronary artery a coefficient of 2.5 is used and for the proximal right coronary artery a coefficient of 1 is used. The exclusion criterion was the presence of noncoronary artery disease-related conditions such as infectious or systematic inflammatory diseases, severe 


\section{Cellular Physiology Cell Physiol Biochem 2017;43:172-181 \begin{tabular}{l|l|l} 
and Biochemistry & $\begin{array}{l}\text { DOI: 10.1159/000480336 } \\
\text { Published } 2017 \text { (2017 The Author(s). Published by S. Karger AG, Basel } \\
\text { www.karger.com/cpb }\end{array}$
\end{tabular}}

Chen et al.: Post-Translational Modification of Adiponectin

renal and hepatic diseases, active malignancy or pregnancy. Clinical characteristics, including demographic data, body weight, height, waist circumference, medical history and medication use, were obtained from the hospital records. Body mass index (BMI) was calculated as weight (kg) divided by the square of height $\left(\mathrm{m}^{2}\right)$. Dislipidemia was defined as both triglyceride $(\mathrm{TG})>1.7 \mathrm{mmol} / \mathrm{L}$ and total cholesterol $(\mathrm{TC})>5.18$ $\mathrm{mmol} / \mathrm{L}$ and HDL-cholesterol (HDL-c) $<1.0 \mathrm{mmol} / \mathrm{L}$. Patients with arterial blood pressure measurements above $140 / 90 \mathrm{mmHg}$ or a history of current antihypertensive drug use were considered hypertensive. The presence of diabetes mellitus (DM) was defined as fasting plasma glucose (FBG) above $126 \mathrm{mg} / \mathrm{dL}$ or a history of actual antidiabetic drug use. The study was approved by the Institutional Ethic Committee of Union Hospital, Huazhong University of Science and Technology.Written, informed consent was obtained from all subjects for collection of the blood samples as well as data required for the study.

\section{ELISA}

Serum total and HMW Apn concentrations were measured by ELISA (47-ADPHU-E01, Alpco, Salem, NH, USA) according to manufacturer's instruction. The dilution factors was 1:5000. Three samples of known concentration were tested twenty times on one plate to assess intra-assay precision and intraassay coefficient of variance (CV) was $3.51 \%$. Three samples of known concentration were tested in forty separate assays to assess inter-assay precision and inter-assay CV was $6.55 \%$.

\section{Detection of glycosylation}

Apn from patients and healthy human plasmas was immunoprecipitated with a mouse-against human monoclonal antibody (ab16086,Abcam, Cambridge, MA, USA) and a polyclonal rabbit anti-mouse antibody conjugated to protein A-Sepharose (Dingguo, Beijing, China). Immunocomplexes were washed 6 times in cold PBS containing $1 \%$ NP40 and $0.1 \%$ SDS after rocking overnight. The immunocomplexes were separated on non-reducing SDS electrophoresis and analyzed by western blots with monoclonal anti-human Apn (R\&D system, Shanghai,China). Total glycoprotein was detected by staining of gels with ProQ Emerald Green 300 (P21857, Molecular ProbesTM, Invitrogen, USA) after treatment with $44 \mathrm{mM}$ metaperiodate (BioUltra, $\geq 99.5 \%$, Sigma-Aldrich, USA). Glycosylated HMW, MMW and LMV Apn markers were purified from a healthy human serum by using gel permeation chromatography. The glycosylation rate of Apn was calculated according to the ratio of glycosylated Apn and the glycosylated Apn marker.

\section{Cell culture and transfection}

The Human VSMCs were obtained from Modern Analysis and Testing Center of Union Hospital, Huazhong University of Science and Technology and cultured in a mixture medium of DMEM/F12 (1:1) adding $100 \mathrm{U} / \mathrm{mL}$ penicillin, $100 \mu \mathrm{g} / \mathrm{mL}$ streptomycin, and $12 \%$ heat inactivated $\mathrm{FBS}$ and incubated at $37^{\circ} \mathrm{C}$ in $5 \% \mathrm{CO}_{2}$. Passages were consecutively performed with the cells grown into a monolayer until generation $5-6$, which were used in the study. Immediately before treatment, the medium was removed, the cells were washed once with PBS and then treated with $40 \mu \mathrm{g} / \mathrm{ml}$ oxLDL (>97\%,1.0 mg/ml; UBC, Brussels Belgium) for $4 \mathrm{~h}$ after pretreatment with $5 \mu \mathrm{g} / \mathrm{ml}$ of glycosylated (catalogue number: CYT-434, $30 \mathrm{kDa}$; Prospec, Israel) or non-glycosylated Apn (catalogue number: CYT-280, $24 \mathrm{kDa}$; Prospec, Israel) or DMSO vehicle for $24 \mathrm{~h}$. Glycosylated Apn was a recombinant human Apn produced in HEK cells and non-glycosylated Apn was a recombinant non-glycosylated human Apn produced in E. Coli. The Apn used in these functional studies was determined as a single subunit one because of the molecular mass.

Cells were seeded into 12-well plates overnight and transiently transfected by siRNAs against AdipoR1 or AdipoR2, or control siRNA (Abm, Inc. Canada) with a MOI of 1:10. Twenty-four hours post-transfection, the cells were treated with $40 \mu \mathrm{g} / \mathrm{ml}$ oxLDL for $4 \mathrm{~h}$ after pretreatment with $5 \mu \mathrm{g} / \mathrm{ml}$ of glycosylated or nonglycosylated Apn for 24h.

Indirect Immunofluorescence assay (IFA)

The VSMCs were fixed, permeabilized and subsequently combined with rabbit anti-adipo R1 or antiadipo R2 antibody was from Abcam (Cambridge, UK). Then, the cells were incubated with FITC-conjugated anti-IgG antibodies (Sigma, USA) in the dark. The cells were mounted and observed on a confocal microscopy (BD,USA).

\section{Determination of cellular total cholesterol}

Cells were lysed and the supernatant was collected after centrifugation at $10000 \mathrm{~g} \times 10 \mathrm{~min}$. Detection reagent $(1000 \mu \mathrm{L})$ was reacted with $50 \mu \mathrm{L}$ of cholesterol standard or collected cell lysate supernatant 
according to manufacturer's instruction (Jixin Biotechnology, China). After $37^{\circ} \mathrm{C} 5 \mathrm{~min}$, absorbance at $520 \mathrm{~nm}$ was measured. A standard curve was prepared using cholesterol standard solution, and the concentrations of cholesterol in the samples were calculated according to the standard curve. The final result was determined in terms of $10^{6}$ cells cholesterol content ( $\left.\mu \mathrm{mol} / 10^{6} \mathrm{cells}\right)$ [18].

\section{Proliferation assay}

VSMCs were replanted into 96-well plates with $10^{4}$ cells /well, and incubated at $37^{\circ} \mathrm{C} 5 \% \mathrm{CO}_{2}$ for 4,8 , $12,24,48$ and $72 \mathrm{~h}$, respectively. Then, Cell Counting Kit (CCK)-8 $(10 \mu \mathrm{l})$ reagent was added to the obtained cells at different time points and incubated for $3 \mathrm{~h}$, then optical density (OD) at $450 \mathrm{~nm}$ was recorded and the cell growth curves were plotted. Four independent experiments were performed.

\section{Analysis of cell migration}

To monitor cell migration in real time, we used the transwell chambers with 24-well inserts (pore size, $8 \mu \mathrm{m}$; Corning Costar, Corning, NY, USA). $10^{5}$ cells were plated in the top chamber and incubated for $24 \mathrm{~h}$. The cells in the top chamber were plated in medium without serum or growth factors, and medium supplemented with serum was used as a chemoattractant in the lower chamber. After $24 \mathrm{~h}$ of incubation, migrated cells through the pores were counted under a light microscope after crystal violet staining. The migration rate was calculated according to the ratio of migrated cells and total cells.

\section{Statistical analysis}

Data are expressed as means \pm standard errors of the means. Analysis was performed using SPSS 15.0 for Windows. Statistical significance was tested using either one-factor analysis of variance if the data consisted of a normal distribution or a rank test if the data were not consistent with a normal distribution. Other statistical analysis is shown in Figure Legends. A $P$ value of $<0.05$ was considered statistically significant.

\section{Results}

\section{Apn released from healthy and CAD patients}

Normal coronary arteries were observed in 15 patients' coronary angiographies (no stenosis). The remaining 77 patients were divided into two groups according to the severity of AS determined by the GS. Patients with GS between 1 to 29 were defined as the mild AS group ( $\mathrm{n}=31$ ), and the patients with GS $\geq 30$ were defined as the severe AS group ( $n=46$ ) (Table 1) . The results showed that dyslipidemias, recognized risk factors for atherosclerotic cardiovascular disease is correlative significantly with progress of stenosis in CAD patients and hypertension is significantly correlative with CAD. In addition, the incidence of DM increased in CAD patients with mild stenosis, and again in severe stenosis.

Table 1. Characteristics of participants. Data shown are mean $\pm \mathrm{SD}$, or n (\%). The relationships between variables were explored by simple correlation and multiple regression analyses. BMI, body mass index; CAD, coronary artery disease; DM, diabetes mellitus. ** $p<0.01$. ${ }^{\text {a }}$ compared to healthy group. ${ }^{b}$ compared to no stenosis. ${ }^{\mathrm{C}}$ compared to mild stenosis group

\begin{tabular}{lcccc}
\hline & Healthy volunteer & & CAD & \\
Independent variables & $\begin{array}{c}\text { No stenosis } \\
(\mathrm{n}=20)\end{array}$ & $\begin{array}{c}\text { Mild } \\
(\mathrm{n}=15)\end{array}$ & $\begin{array}{c}\text { stenosis } \\
(\mathrm{n}=31)\end{array}$ & $\begin{array}{c}\text { Severe } \\
\text { stenosis } \\
(\mathrm{n}=46)\end{array}$ \\
\hline Age (years) & $56.4 \pm 6.1$ & $54.6 \pm 4.2$ & $58.4 \pm 3.9$ & $59.4 \pm 3.8$ \\
Male & $9(45.0)$ & $7(46.6)$ & $16(51.6)$ & $24(52.2)$ \\
Female & $11(55.0)$ & $8(53.4)$ & $15(47.4)$ & $22(47.8)$ \\
BMI (kg/m $)$ & $27.2 \pm 3.4$ & $29.8 \pm 2.2$ & $28.34 \pm 2.37$ & $29.02 \pm 2.61$ \\
Dyslipidemia & $7(35.0)$ & $8(53.3)$ & $24(77.4)^{* *} \mathrm{a}, \mathrm{b}$ & $38(82.6)^{* *} \mathrm{a}, \mathrm{b}$ \\
TG (mM) & $1.53 \pm 1.01$ & $1.63 \pm 1.02$ & $1.66 \pm 0.93$ & $1.77 \pm 1.12$ \\
TC (mM) & $4.32 \pm 0.85$ & $4.41 \pm 0.63$ & $4.55 \pm 0.91$ & $4.63 \pm 1.05$ \\
HDL-c (mM) & $1.25 \pm 0.36$ & $1.09 \pm 0.53$ & $1.11 \pm 0.63$ & $1.03 \pm 0.65$ \\
DM (\%) & $0(0)$ & $0(0)$ & $7(22.6)^{* *} \mathrm{a}, \mathrm{b}$ & $21(45.7)^{* *} \mathrm{a}, \mathrm{b}, \mathrm{c}$ \\
FBG (mM) & $4.9 \pm 1.03$ & $5.36 \pm 1.11$ & $6.31 \pm 0.88 \mathrm{a}$ & $6.57 \pm 1.39 \mathrm{a}$ \\
Hypertension (\%) & $0(0)$ & $12(80.0)^{* *} \mathrm{a}$ & $26(83.9)^{* *} \mathrm{a}$ & $39(84.8)^{* * a}$ \\
Total Apn & $15.1 \pm 1.59$ & $16.3 \pm 1.23$ & $18.5 \pm 1.04$ & $27.7 \pm 1.36^{* *} \mathrm{a}, \mathrm{b}, \mathrm{c}$ \\
HMV Apn & $9.93 \pm 0.84$ & $8.22 \pm 0.85$ & $9.14 \pm 1.01$ & $15.5 \pm 0.79^{* *} \mathrm{a}, \mathrm{b}, \mathrm{c}$ \\
\hline
\end{tabular}




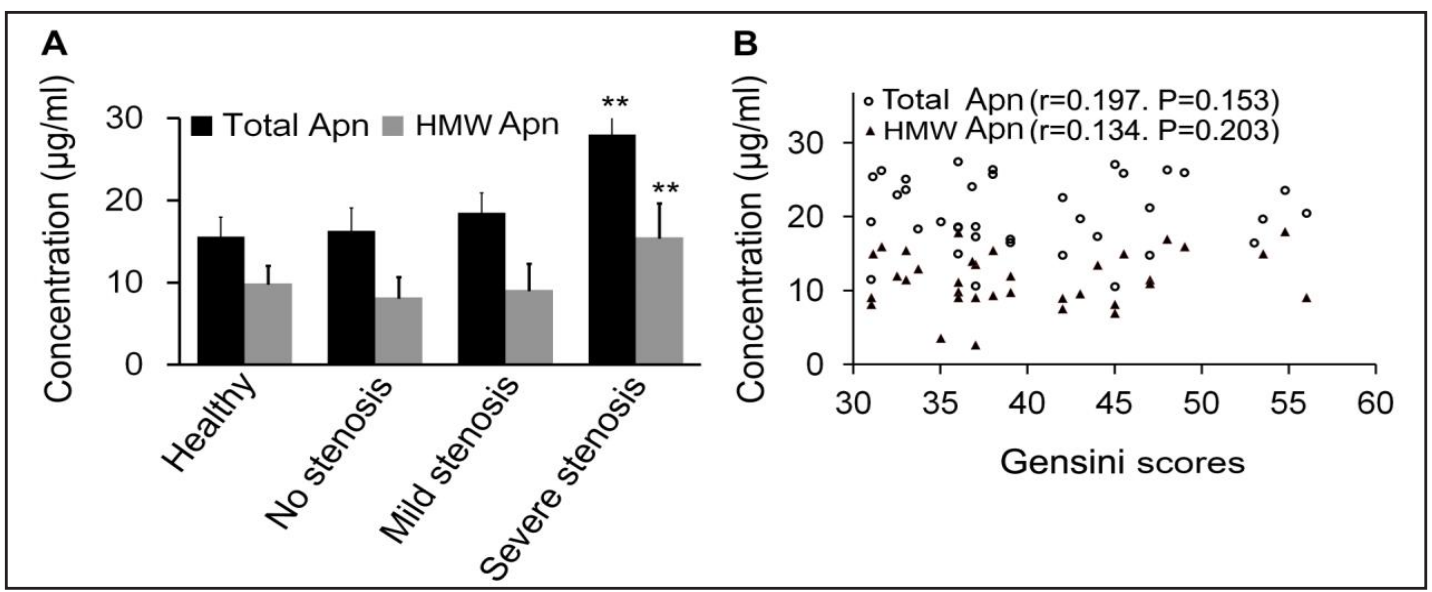

Fig. 1. Circulating and HMW Apn levels and underlying cardiovascular disease status. (A) the total or HMW Apn levels increased in severe stenosis groups; (B) there was no association between total Apn or HMW Apn levels and GS of CAD patients in severe stenosis group $(n=46) .{ }^{* *} \mathrm{P}<0.01$ vs Healthy volunteers.

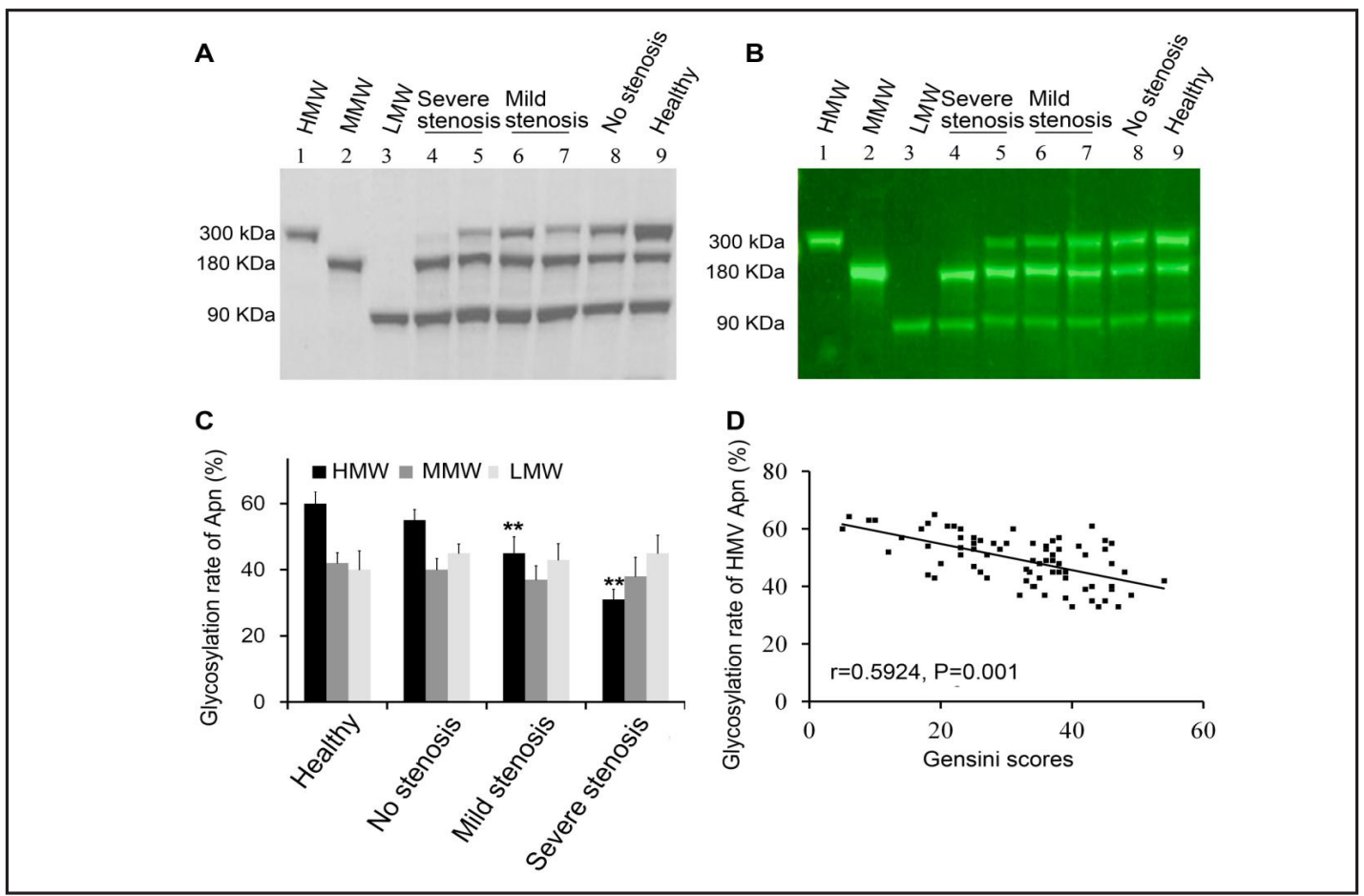

Fig. 2. Glycosylation rate of HMW Apn in the collagenous domain decreased in AS patients. (A) Western blot identification of Apn on non-reducing SDS gel electrophoresis. (B) Glycosylation of Apn was detected by ProQ Emerald 300 after treatment with 44 mM metaperiodate. Lane 1-3 represent HMW, MMW and LMW Apn markers respectively; lane 4-5 represent severe stenosis; lane 6-7 represent mild stenosis; lane 8 represent no stenosis and lane 9 represent healthy volunteer. (C) Glycosylation of Apn in different group ( ${ }^{* *} \mathrm{P}<0.01$ vs. healthy group). Glycosylation of HMW Apn decreased in mild and severe stenosis. (D) Correlation between glycosylation rate of HMW Apn and GS of CAD patients. The glycosylation rate of Apn was calculated according to the ratio of glycosylated Apn and total Apn in the bands.

Statistical results showed that there was a striking elevation of the total or HMW Apn in severe stenosis (Fig. 1A). Pearson correlation analyses showed that there was no significant correlation between the total or HMW Apn levels and GS of CAD patients (Fig. 1B). 


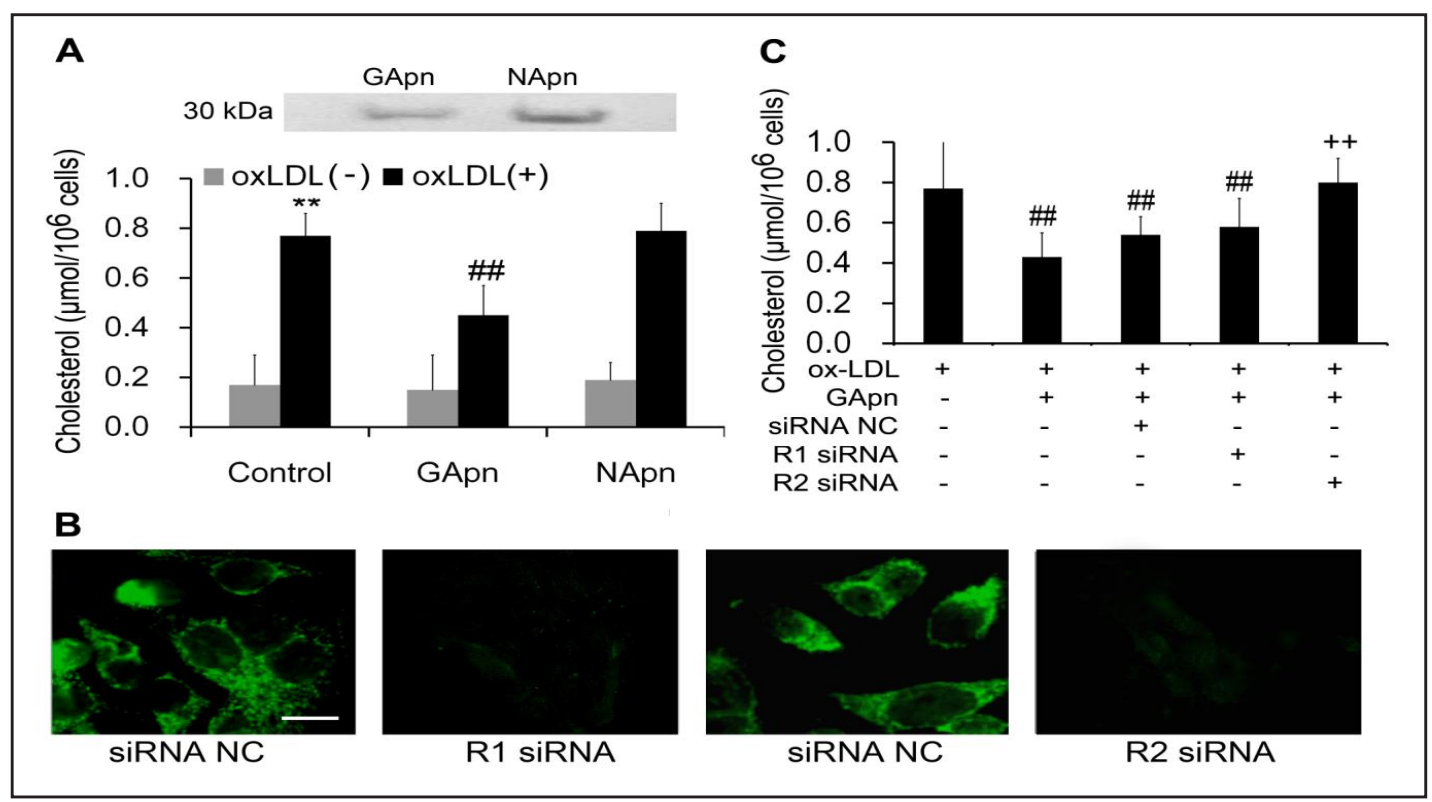

Fig. 3. The total cholesterol contents were assayed by cholesterol oxidase reaction $(n=6, * * P<0.01 v s$. oxLDL (-) control; \#\# P <0.01 vs. oxLDL (+) control; ++ P <0.01 vs. oxLDL (+), GApn (+)). A: Verification of GApn and NApn by western blot (upper panel) and the effects of APN on cholesterol accumulation (lower panel); B: The inhibitory efficiencies of siRNA were assayed by IFA (Scale bar: $10 \mu \mathrm{m}$ ); C: The effects of adipoR1 siRNA or adipoR2 siRNA on cholesterol accumulation. The results showed that GApn inhibited cholesterol accumulation induced by oxLDL in VSMCs, which was abrogated by adipoR2 siRNA. GApn, Glycosylated Apn; NApn, non-glycosylated Apn.

\section{Glycosylation rate of HMV Apn in the collagenous domain decreased in AS patients}

The Apn in the blood sample was immunoprecipitated and $50 \mu \mathrm{g}$ of immunocomplex was separated on non-reducing SDS electrophoresis. The expression of Apn was identified by Western blot (Fig. 2A). The presence of glycosylation on human Apn was confirmed by the staining of polyacrylamide gels with ProQ Emerald 300 stain after oxidation with $44 \mathrm{mM}$ metaperiodate (Fig. 2B). The results showed that glycosylation of HMW decreased in CAD patients with mild and severe stenosis when compared with healthy volunteers (Fig. 2C). Correlation analyses also showed that there was significantly a negative correlation between glycosylation levels of HMW and GS scores of CAD ( $F=-14.69, P=0.001$ ) when compared with healthy volunteers (Fig. 2D).

\section{Glycosylated Apn inhibited accumulation of total cholesterol in VSMCs}

VSMCs were harvested after administration of glycosylated or non-glycosylated Apn (Fig. $3 \mathrm{~A}$ ) with or without oxLDL treatment and rinsed twice with PBS (pH 7.2, 0.01 M). Then cells were lysed by RIPA cell lysis reagent, and the supernatants were collected for total cholesterol content assay. The results showed that the cholesterol contents of the cells significantly increased in oxLDL treated cells. After administration of glycosylated Apn, the total cholesterol contents in VSMCs induced by oxLDL were significantly decreased, while not in non-glycosylated Apn group (Fig. $3 \mathrm{~A}$ ). To investigate which Apn receptor was involved in the action, siRNAs against AdipoR1 or AdipoR2 were transfected into VSMCs, and the inhibitory efficiencies were verified by IFA (Fig. 3 B). The inhibitory effects of glycosylated Apn on cholesterol accumulation were abrogated by adipoR2 siRNA (Fig. 3 C), indicating that glycosylated Apn controlled the balance of cholesterol metabolism in VSMCs and inhibited intracellular cholesterol accumulation through adipoR2 . 


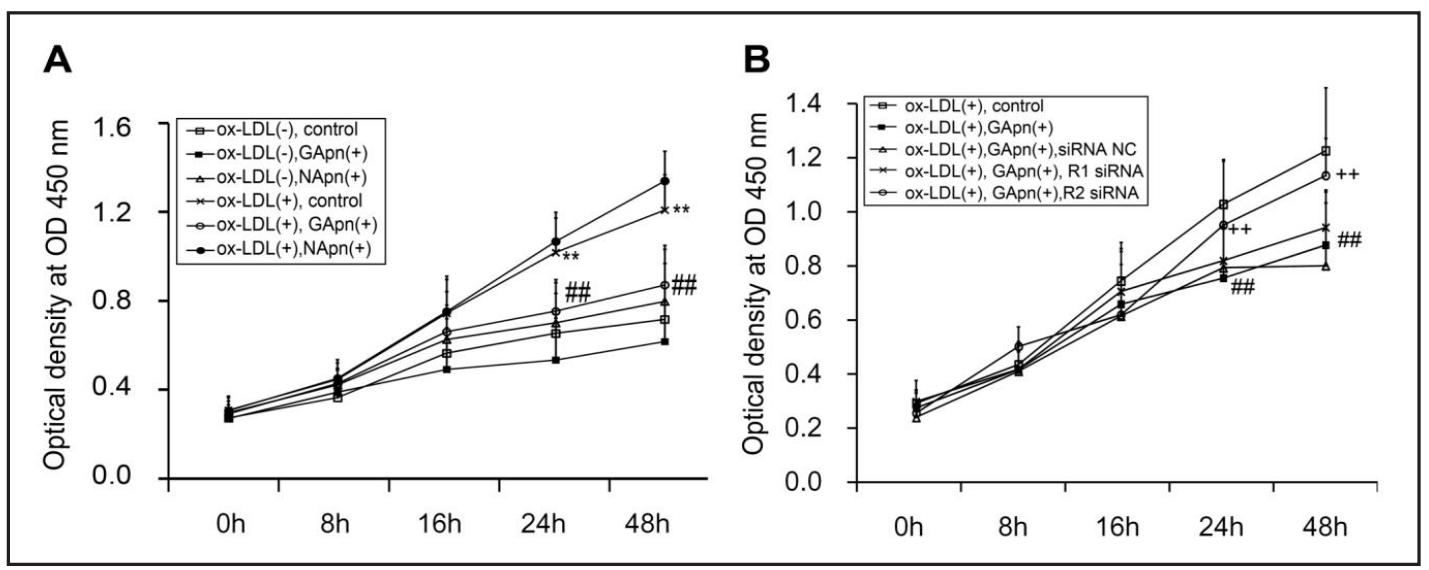

Fig. 4. The proliferation of VSMCs was assayed by CCK-8 kit ( $\mathrm{n}=5$, ** $\mathrm{P}<0.01$ vs.oxLDL (-) control; \#\# $\mathrm{P}<0.01$ vs. oxLDL (+) control; ++ P $<0.01$ vs. oxLDL (+), GApn (+)). The results showed that glycosylated Apn inhibited the proliferation of oxLDL-treated VSMCs (A), which was abrogated by adipoR2 siRNA (B). GApn, Glycosylated Apn; NApn, non-glycosylated Apn.

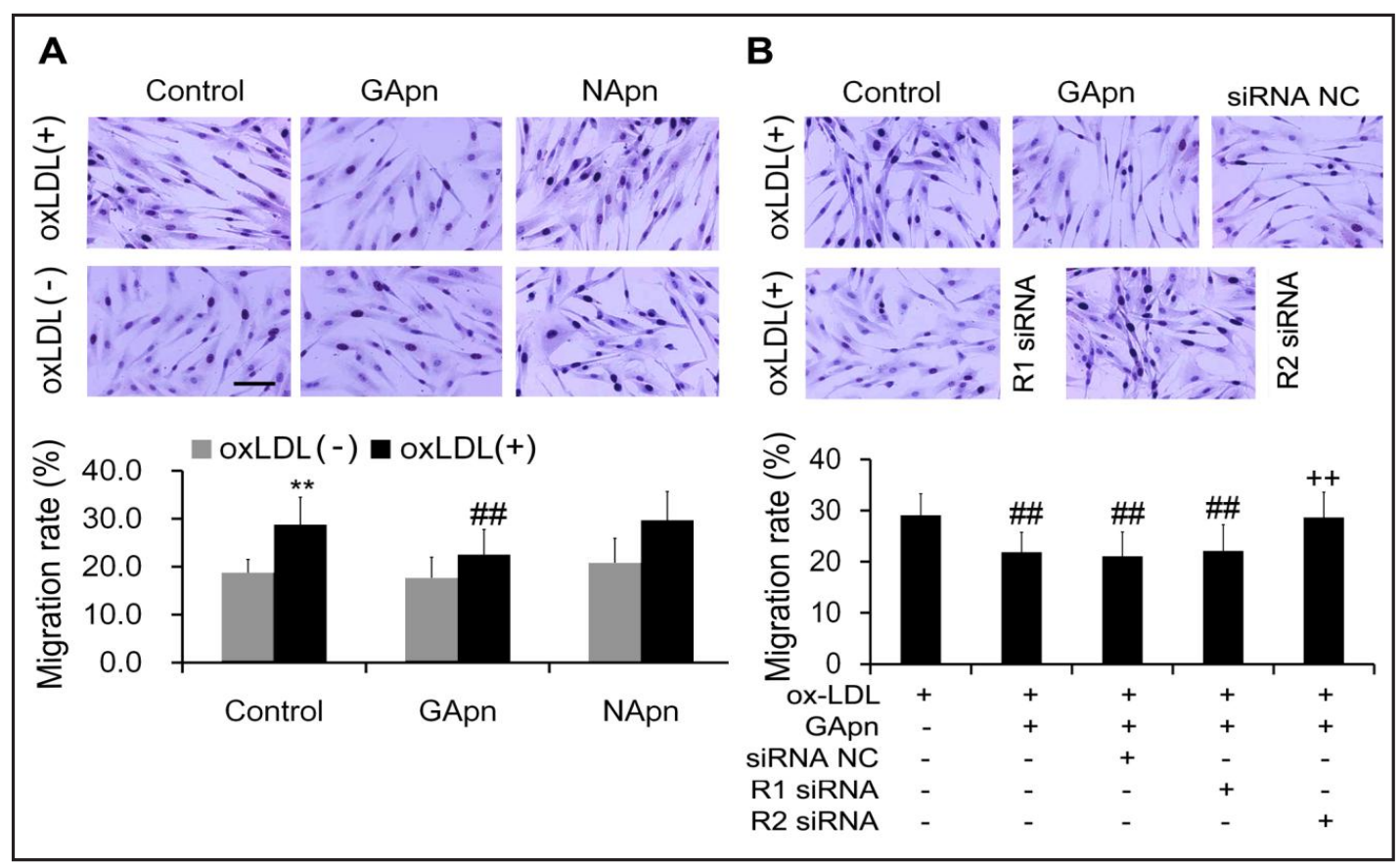

Fig. 5. The migration of VSMCs was assayed by transwell chambers ( $n=5, * * P<0.01 v s$. oxLDL ( - ) control; \#\# P<0.01 vs. oxLDL (-) control; ++ P <0.01 vs. oxLDL (+), GApn (+). The results showed glycosylated Apn inhibited the migration of oxLDL-treated VSMCs (A, scale bar: $50 \mu \mathrm{m}$ ), which was abrogated by adipoR2 siRNA (B). GApn, Glycosylated Apn; NApn, non-glycosylated Apn. The migration rate was calculated according to the ratio of migrated cells and total cells.

\section{Glycosylated Apn inhibited proliferation of oxLDL-injuried VSMCS}

The results of the CCK-8 assay showed the cell proliferation of normal VSMCs was not affected by glycosylated or non-glycosylated Apn. However, glycosylated Apn inhibited the proliferation of oxLDL-treated control VSMCs, which increased significantly when compared with oxLDL-untreated control VSMCs (Fig. 4 A). The inhibitory effects of glycosylated Apn on 
proliferation of oxLDL-treated VSMCs were abrogated by adipoR2 siRNA (Fig. 4 B), indicating that glycosylated Apn inhibited proliferation of oxLDL-treated VSMCs through adipoR2.

\section{Glycosylated Apn inhibited migration of oxLDL-stressed VSMCS}

The results of the migration assay showed that the cell migration rates were not affected by glycosylated or non-glycosylated Apn on oxLDL-untreated control VSMCs, however, the cell migration rates were significantly inhibited by glycosylated Apn on oxLDL-treated VSMCs, which increased significantly when compared with oxLDL-untreated control VSMCs (Fig. 5 A). The inhibitory effects of glycosylated Apn on migration of oxLDL-treated VSMCs were abrogated by adipoR2 siRNA (Fig.5B), indicating that glycosylated Apn inhibited migration of oxLDL-treated VSMCs through adipoR2.

\section{Discussion}

Despite its potential antiatherogenic effects, circulating Apn has not been established as a biomarker in cardiovascular disease (CVD) because of the conflicting data on its clinical predictive value [19]; although the first evidence supported that higher Apn predicts low CVD risk in healthy individuals, elevated Apn levels have been associated with increased mortality in patients with ischemic heart disease [19]. Moreover, in recent meta-analyses, circulating Apn has been associated with increased risk for stroke and higher risk for CVD and total mortality in secondary prevention [19]. In the present study, the detection of blood samples from CAD patients and healthy individuals showed that although there was a striking elevation of circulating or HMW Apn in severe stenosis, there was no significant correlation between circulating or HMW Apn levels and GS of CAD patients. Different isoforms of Apn may activate different signaling pathways and have differing effects in a range of tissues [20, 21]. In the present study, the detection of glycosylation of Apn revealed that glycosylation of HMW Apn decreased in CAD patients with mild and severe stenosis, and there was significant correlation between glycosylated levels of HMW Apn and GS of CAD patients, indicating that the glycosylation of HMW affected the function of Apn in human plasma. This conclusion is supported by a study of unseparated plasma Apn in type 2 diabetic patients, in whom impaired multimerization of human adiponectin mutants associated with diabetes [22].

Using oxidized-injured human VSMCs, we set out to determine whether and how posttranslational Apn is involved in the total cholesterol accumulation in cells. We verified that only glycosylated Apn, not non-glycosylated Apn inhibited cholesterol accumulation induced by oxLDL in VSMCs through the adipoR2 receptor. Further, we verified that glycosylated Apn inhibited proliferation and migration of oxLDL-treated VSMCs through adipoR2.

Besides, glycosylated HMW Apn concentrations from CAD patients reduced compared with those from Healthy volunteers and have few changes between CAD groups. Nonglycosylated HMW Apn levels from Mild and Severe stenosis increase gradually depend on its severity compared with Healthy group. Thus, it was concluded that 1) glycosylated HMW Apn could be a positive/healthy factor against CAD since it reduced in CAD patient. 2) nonglycosylated HMW Apn might be a pathophysiological factor in CAD since it increased in CAD patients who have mild or severe stenosis.

\section{Conclusion}

These findings confirm that Apn derived from blood samples of AS patients with severe stenosis significantly increased, however the glycosylation rate of HMW Apn significantly decreased. Non-glycosylated HMW Apn might cancel the inhibitory effects of Apn on lipid accumulation and proliferation and migration of VSMCs induced by oxidized LDL. Taken together, these data suggest that glycosylation of HMW Apn is critically involved in regulating the function against AS by inhibiting lipid accumulation and proliferation and migration of VSMCs. 


\section{Cellular Physiology Cell Physiol Biochem 2017;43:172-181

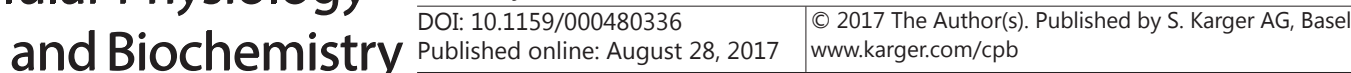

Chen et al.: Post-Translational Modification of Adiponectin

\section{Acknowledgements}

This work wassupported by the National Natural Science Fund ofChina (Grants 81270265, 30872541) and the Applied Basic Research Programs of Wuhan (No.2014060101010031).

\section{Disclosure Statement}

The authors have declared that no competing interests exist.

\section{References}

1 Wang Y, Lam KS, Chan L, Chan KW, Lam JB, Lam MC, Hoo RC, Mak WW, Cooper GJ, Xu A: Post-translational modifications of the four conserved lysine residues within the collagenous domain of Apn are required for the formation of its high molecular weight oligomeric complex. J Biol Chem 2006;281:16391-16400.

2 Wang Y, Lu G, Wong WP, Vliegenthart JF, Gerwig GJ, Lam KS, Cooper GJ, Xu A: Proteomic and functional characterization of endogenous Apn purified from fetal bovine serum. Proteomics 2004;4:3933-3942.

3 Tsao TS: Assembly of Apn oligomers. Rev Endocr Metab Disord 2004;15:125-136.

4 Wang Y, Xu A, Knight C, Xu LY, Cooper GJ: Hydroxylation and glycosylation of the four conserved lysine residues in the collagenous domain ofApn. Potential role in the modulation of its insulin-sensitizing activity. J Biol Chem 2002;277:19521-19529.

5 Takuwa A, Yoshida T, Maruno T, Kawahara K, Mochizuki M, Nishiuchi Y, Kobayashi Y, Ohkubo T: Ordered self-assembly of the collagenous domain of Apn with noncovalent interactions viaglycosylated lysine residues. FEBS Lett 2016;590:195-201.

-6 Jungtrakoon P, Plengvidhya N, Tangjittipokin W, Chimnaronk S, Salaemae W, Chongjaroen N, Chanprasert K, Sujjitjoon J, Srisawat C, Yenchitsomanus PT: Novel Apn variants identified in type 2 diabetic patients reveal multimerization and secretion defects. PLoS One 2011;6:e26792.

7 Liu X, Perakakis N, Gong H, Chamberland JP, Brinkoetter MT, Hamnvik OR, Mantzoros CS: Apn administration prevents weight gain and glycemic profile changes in diet-induced obese immune deficient Rag1-/- mice lacking mature lymphocytes. Metabolism 2016;65:1720-1730.

-8 Freitas Lima LC, Braga VA, do Socorro de França Silva M, Cruz JC, Sousa Santos SH, de Oliveira Monteiro MM, Balarini CM: Adipokines, diabetes and atherosclerosis: an inflammatory association. Front Physiol 2015;6: 04.

-9 Gao Q Yao X, Zheng J: MiR-323 Inhibits Prostate Cancer Vascularization Through Adiponectin Receptor. Cell Physiol Biochem 2015;36:1491-1498.

10 Zeng F, Shi J, Long Y, Tian H, Li X, Zhao AZ, Li RF, Chen T: Adiponectin and Endometrial Cancer: A Systematic Review and Meta-Analysis. Cell Physiol Biochem 2015;36:1670-1678.

11 Li L, Liu M, Kang L, Li Y, Dai Z, Wang B, Liu S, Chen L, Tan Y, Wu G: HHEX: A Crosstalker between HCMV Infection and Proliferation of VSMCs. Front Cell Infect Microbiol 2016;6:169.

$\checkmark 12$ Matsuda M, Shimomura I: Roles of Apn and oxidative stress in obesity-associated metabolic and cardiovascular diseases. Rev Endocr Metab Disord 2014;15:1-10.

13 Kitagawa K, Miwa K, Okazaki S, Sakaguchi M, Mochizuki H: Serum high-molecular-weight Apn level and incident dementia in patients with vascular riskfactors. Eur J Neurol 2016;23:641-647.

-14 Hascoet S, Elbaz M, Bongard V, Bouisset F, Verdier C, Vindis C, Genoux A, Taraszkiewicz D, Perret B, Galinier M, Carrié D, Ferrières J, Ruidavets JB: Apn and long-term mortality in coronary artery disease participants and controls. Arterioscler Thromb Vasc Biol 2013;33:e19-29.

15 Satoh M, Tabuchi T, Minami Y, Takahashi Y, Itoh T, Nakamura M: Prospective, randomized, single-blind comparison of effects of 6 months of treatment with telmisartan versus enalapril on high-molecularweight Apn concentrations in patients with coronary artery disease. Clin Ther 2009;31:2113-2125.

16 von Eynatten M, Humpert PM, Bluemm A, Lepper PM, Hamann A, Allolio B, Nawroth PP, Bierhaus A, Dugi KA: High-molecular weight Apn is independently associated with the extent of coronary artery disease in men. Atherosclerosis 2008;199:123-128. 


\section{Cellular Physiology Cell Physiol Biochem 2017;43:172-181

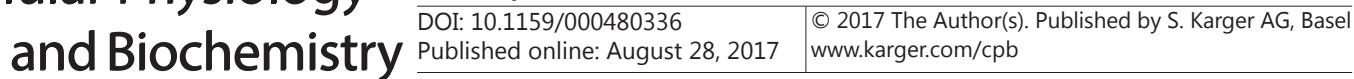 \\ Chen et al.: Post-Translational Modification of Adiponectin}

-17 Pothineni NV, Rochlani Y, Vallurupalli S, Kovelamudi S, Ahmed Z, Hakeem A, Mehta JL: Comparison of Angiographic Burden of Coronary Artery Disease in Patients With Versus Without Hepatitis C Infection. Am J Cardiol 2015;116:1041-1044.

18 Li L, Li Y, Dai Z, Liu M, Wang B, Liu S, Wang L, Chen L, Tan Y, Wu G: Lipid metabolism in vascular smooth muscle cells infuenced by HCMV Infection. Cell Physiol Biochem 2016; 39:1804-1812.

19 Sook Lee E, Park SS, Kim E, Sook Yoon Y, Ahn HY, Park CY, Ho Yun Y, Woo Oh S: Association between Apn levels and coronary heart disease and mortality: a systematic review and meta-analysis. Int J Epidemiol 2013;42:1029-1039.

-20 Tsao TS, Tomas E, Murrey HE, Hug C, Lee DH, Ruderman NB, Heuser JE, Lodish HF: Role of disulfide bonds in Acrp30/Apn structure and signaling specificity. Different oligomers activate different signal transduction pathways. J Biol Chem 2003;278:50810-50817.

-21 Neumeier M, Weigert J, Schäffle, A, Wehrwein G, Müller-Ladner U, Schölmerich J, Wrede C, Buechler C: Different effects of Apn isoforms in human monocytic cells. J Leukoc Biol 2006;79:803-808.

22 Waki H, Yamauchi T, Kamon J, Ito Y, Uchida S, Kita S, Hara K, Hada Y, Vasseur F, Froguel P, Kimura S, Nagai $\mathrm{R}$, Kadowaki T: Impaired multimerization of human adiponectin mutants associated with diabetes. Molecular structure and multimer formation of adiponectin. J Biol Chem 2003;278:40352-40363. 